\title{
BMJ Open Antimicrobial-associated organ injury among the elderly: a systematic review and meta-analysis protocol
}

\author{
Tichawona Chinzowu (D) , ${ }^{1}$ Sandipan Roy, ${ }^{2}$ Prasad S Nishtala ${ }^{3}$
}

To cite: Chinzowu T, Roy S, Nishtala PS. Antimicrobialassociated organ injury among the elderly: a systematic review and metaanalysis protocol. BMJ Open 2022;12:e055210. doi:10.1136/ bmjopen-2021-055210

- Prepublication history and additional supplemental material for this paper are available online. To view these files, please visit the journal online (http://dx.doi.org/10.1136/ bmjopen-2021-055210).

Received 05 July 2021 Accepted 28 January 2022

A) Check for updates

(c) Author(s) (or their employer(s)) 2022. Re-use permitted under CC BY-NC. No commercial re-use. See rights and permissions. Published by BMJ.

${ }^{1}$ Pharmacy and Pharmacology, University of Bath, Bath, UK

${ }^{2}$ Department of Mathematical Science, University of Bath, Bath, UK

${ }^{3}$ Department of Pharmacy and Pharmacology, University of Bath, Bath, UK

Correspondence to Mr Tichawona Chinzowu; tc888@bath.ac.uk

\begin{abstract}
Introduction Older adults (aged 65 years and above) constitute the fastest growing population cohort in the western world. There is increasing evidence that the burden of infections disproportionately affects this cohort of older adults and hence this vulnerable population is frequently exposed to antimicrobials. There is currently no systematic review summarising the evidence for risk of organ injury following antimicrobial exposure among older adults. This protocol will outline how we will conduct a systematic review and meta-analyses to examine the relationship between antimicrobial exposure and organ injury in older adults.
\end{abstract}

Methods and analysis We will search for PsycINFO, PubMed and EMBASE databases for relevant articles using MeSH terms where applicable. After removing duplicates, articles will be screened for inclusion into or exclusion from the study by two reviewers. Title and abstract screening will be done first, followed by full-text screening. The Newcastle-0ttawa scale will be used to assess the risk of bias for cohort and case control studies, and the Cochrane collaboration's risk of bias tool will be used for randomised control trials. We will explore the potential sources of heterogeneity and bias using funnel and forest plots of the included studies.

Ethics and dissemination During the conduct of the review, ethical principles will be observed to ensure integrity. Any potential conflicts of interests will be declared, all contributors acknowledged and no plagiarised material will be included in the review.

The systematic review and meta-analysis will be submitted for publication in a peer-reviewed journal in geriatrics. The findings will also be presented at international conferences in geriatrics or pharmacoepidemiology. The results will be communicated to patient and public engagement networks supported by the NHS Research and Development.

PROSPERO registration number This protocol is registered in the PROSPERO database (registration number CRD42020152621).

\section{BACKGROUND}

Older adults aged 65 years and above comprise the fastest and largest expanding population age group in the developed world. ${ }^{1}$ This age group is more prone to infectious diseases such as pneumonia, skin and soft tissue infections, urinary tract infections and septicaemia, when compared
Strengths and limitations of this study

This is the first systematic review and meta-analysis to explore the association of antimicrobial chemotherapy and organ injury among the older adults.

- Selection of studies, data extraction and assessment of bias will be done independently by two researchers, and the third researcher's opinion sought for when there is dispute, thus ensuring that all relevant studies are included without personal bias.

- Only articles published in English will be included, therefore, language bias may exist.

- Causality may be difficult to establish since most studies are expected to be observational.

with other age groups. ${ }^{1}$ It is estimated that the older adults comprise $48.7 \%$ of individuals who are admitted to hospital intensive care units for these infections, ${ }^{2}$ resulting in their increased length of hospital stay and exposure to antibiotics. Giarratano et $a \hat{l}$ highlighted several predisposing factors that make older adults more susceptible to antimicrobial adverse events. These include physiological changes, higher comorbidities, drug-drug interactions, drug delivery routes used and length of time they are in contact or exposed to the antimicrobial agents. In one large study, antimicrobial-related adverse events accounted for $19.3 \%$ of all drug-related adverse events seen at the emergency department. $^{2} \quad$ Several antimicrobial-associated adverse events become apparent several years after drug has been approved and the adverse events reported in clinical trials differ considerably from post marketing surveillance. Since most trials exclude older adults, the true nature and incidence of antimicrobialrelated adverse events in this population are unknown. In their review, Giarratano et $a \hat{P}$ concluded that there is a general lack of epidemiological studies on antimicrobials used among the older adults, yet this is essential in informing healthcare providers to achieve optimal safety and effectiveness when 
providing antimicrobial pharmacotherapy to the older adults. At the age of 80 years, renal clearance is reduced to about $50 \%$ when compared with age of 30 years. ${ }^{4}$ Hepatic clearance is also reduced by up to $40 \%$ among the elderly due to reduced drug metabolism using the cytochrome P-450 system. ${ }^{5}$ According to Werth, ${ }^{6}$ certain antimicrobials like fluoroquinolones are associated with tissue injury such as tendinopathy. ${ }^{6}$ A systematic review is much needed to synthesise the evidence given the potential for public health implications if antimicrobials are associated with organ injury in older adults. This paper describes a protocol for carrying out a systematic review and meta-analysis to investigate whether antimicrobial exposure is associated with kidney, liver or tissue injury among older adults.

\section{METHODS AND DESIGN}

This protocol was designed following the Preferred Reporting Items for Systematic Reviews and Meta-analyses (PRISMA) guidelines. ${ }^{7}$ The PRISMA-Protocol checklist ${ }^{8}$ was used to guide the presentation of the items of this protocol (see online supplemental file 1).

\section{Review question}

In this protocol, the procedures for a systematic review and meta-analysis that is intended to answer the question: 'Is antimicrobial exposure among the over 65 associated with kidney, liver or tissue injury?' have been outlined.

The components of the population, exposure, comparator and outcome (PECO) are as follows:

- Population: Older adults aged 65 years and above.

- Exposure: Any antimicrobial treatment (due to antibiotics), or long-term antimicrobial treatment (more than 7 days of continuous treatment), or broadspectrum antimicrobial treatment (targeting both Gram-negative and Gram-positive bacteria).

- Comparator: No antimicrobial treatment, or shortterm antimicrobial treatment ( 7 days or less of treatment), or narrow-spectrum antimicrobial treatment (targeting either Gram-negative or Gram-positive bacteria only).

- Outcome: Acute kidney injury, drug-induced liver injury or tissue injury (eg, tendonitis) attributed to the antimicrobial and sustained during or soon after antimicrobial exposure.

\section{Eligible study designs}

This study will include all primary epidemiological observational studies, including cross-sectional studies, prospective cohort studies, retrospective cohort studies, case-control studies and interventional studies that meet the inclusion criteria. However, very few randomised controlled trials (RCTs) are expected to be included in this study. Case reports, case series, reviews, commentaries, editorials, will not be included in this study.

\section{Eligible participants}

A study is eligible for this review if it reported exposure to antimicrobial agents among older adults of 65 years or above. Observational and interventional studies are eligible for inclusion in this study. However, studies reporting antimicrobial exposure to children or adults under 65 years of age will be excluded. Studies reporting exposure of any age group to other medicines but not antimicrobials will also be excluded.

\section{Exposures of interest}

The exposure of interest is the therapeutic use of antimicrobials of any dose, class/type (narrow or broad spectrum), duration or route given for any indication. The comparator is either a group that did not receive any antimicrobials or a group that received an antimicrobial or group of antimicrobials other than the exposure of interest.

\section{Outcome measures of interest}

The main outcome of this study is to determine whether antimicrobial exposure increases organ (liver, kidney or tissue) injury following any antimicrobial exposure among the older adults who are 65 years and above. The main measure of effect will be the relative risk ${ }^{9}$ of organ injury among the exposed when compared with the unexposed populations. Incidence rate of organ (kidney, liver or tissue) injury among the antimicrobial exposed group of the older adults will be compared with the respective incidence rate of the control groups (not exposed to antimicrobials) and expressed as risk ratios.

\section{Additional outcome(s)}

Additional outcomes will include the determination of whether broad spectrum antimicrobials increase the risk of organ injury when compared with narrow spectrum, and whether prolonged exposure increases the risk when compared with short term exposure. The main measure of effect on both outcomes is the risk difference ${ }^{10}$ between comparison groups. The risk of organ injury among participants who received broad spectrum antimicrobials will be compared with those who received narrow spectrum antibiotics. Second, the risk of organ injury among participants with prolonged exposure to antimicrobials will be compared with those on short term. Risk difference will be the main measure of effect on both cases.

\section{Search methods and the identification of studies}

Searches will be conducted in PubMed, Embase, PsycINFO and other databases. Restrictions to publication dates will be applied and limited to the English language. The full search strategy is described in online supplemental file 2.

\section{Selection of eligible studies}

Search results from all databases will be uploaded into EndNote V.X9 ${ }^{11}$ bibliographic software and manually deduplicated. After removing duplicates, studies will be uploaded into Covidence ${ }^{12}$ for the title and abstract screening, as well as full-text screening. Studies identified 


\section{Guide to inclusion and exclusion of studies into the systematic review}

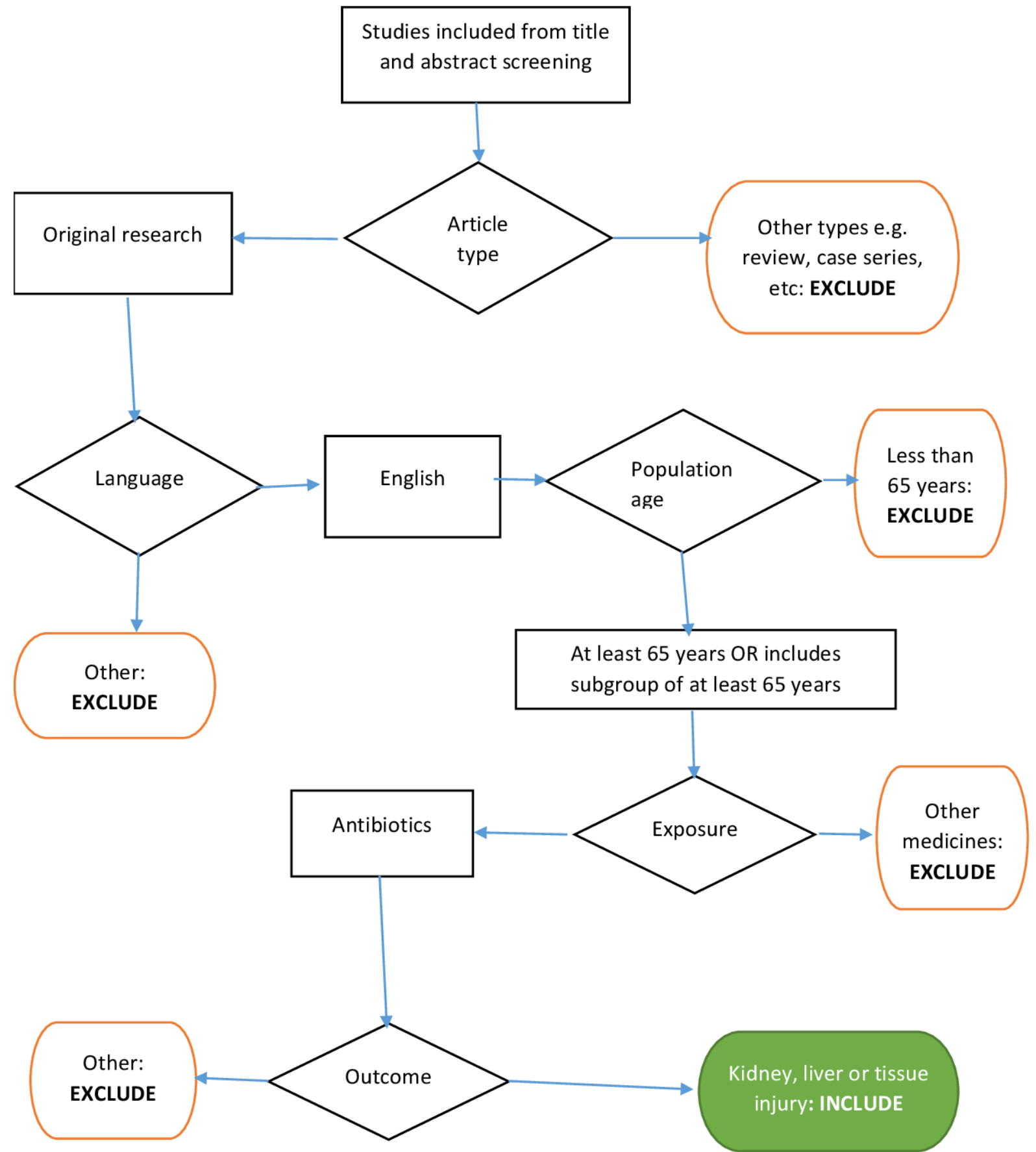

Figure 1 Process flow chart for full-text inclusion of studies into the systematic review from studies selected from the title and abstract screening process.

by the search strategy will be screened independently by two reviewers (TC and PSN) using the titles and/or abstracts for their potential to meet the inclusion criteria described above. The full texts of potentially relevant studies will be retrieved and individually reviewed for eligibility using a standardised assessment tool. A decision process flow chart (figure 1) has been developed to standardise full-text selection by both reviewers. Where the two reviewers disagree, the opinion of the third reviewer (SR) will be sought. Further study information will be sought from the study authors about any missing data or clarification about study participants and study outcomes. 


\section{Data extraction process}

The data to be extracted include study name/article title, authors, journal and full reference, country, study design and setting, participant demographics, exposures (types of antimicrobials including dose, duration and route), sample size of exposed and comparator groups, confounders, statistical methods, primary outcome, secondary outcomes, and participant missing data and reason for missing. Extracted participant demographics for exposure and control groups will include mean or median age at antimicrobial administration, sex and ethnicity. For the primary or secondary study endpoints, wherever applicable, we will extract mean (plus SD), median (plus IQR), number of cases and relative risks (with $95 \%$ CIs).

\section{Risk of bias assessment for eligible studies}

Each reviewer will critically appraise each study and perform the risk of bias using the appropriate tool. Risk of bias for RCTs will be assessed using version 2 of the Cochrane risk-of-bias tool for randomised trials. ${ }^{13}$ In contrast, the Newcastle-Ottawa tool ${ }^{14}$ will be used to assess the risk of bias for observational studies. The quality assessment across the studies will be done using the Grading of Recommendations, Assessment, Development and Evaluation (GRADE) tool ${ }^{15}$ for each outcome. Where the two reviewers differ in opinion, the third reviewer's opinion will be sought. The authors will be contacted for additional information in case of missing or unequivocal information. The following domains will be assessed for the risk of bias:

\begin{tabular}{|c|c|c|}
\hline Type of bias & Type studies & Low/High risk \\
\hline \multirow[t]{2}{*}{$\begin{array}{l}\text { Selection } \\
\text { bias }\end{array}$} & $\begin{array}{l}\text { Controlled } \\
\text { studies }\end{array}$ & $\begin{array}{l}\text { Low risk if random sequence } \\
\text { generation allocation } \\
\text { concealment used }\end{array}$ \\
\hline & $\begin{array}{l}\text { observational } \\
\text { studies }\end{array}$ & $\begin{array}{l}\text { Low risk if patients enrolled as } \\
\text { consecutively observed based on } \\
\text { the pre-existing protocol } \\
\text { Low risk if numbers and reasons } \\
\text { for exclusions were reported } \\
\text { High risk when the association } \\
\text { between exposure and outcome } \\
\text { is different for study participants } \\
\text { compared with non-participants }\end{array}$ \\
\hline \multirow[t]{2}{*}{$\begin{array}{l}\text { Performance } \\
\text { bias }\end{array}$} & $\begin{array}{l}\text { Controlled } \\
\text { studies }\end{array}$ & $\begin{array}{l}\text { High risk if study personnel } \\
\text { were not blinded as to which } \\
\text { intervention the elderly patient } \\
\text { has received }\end{array}$ \\
\hline & $\begin{array}{l}\text { Observational } \\
\text { studies }\end{array}$ & $\begin{array}{l}\text { High risk if there are systematic } \\
\text { differences in the treatment of } \\
\text { participants }\end{array}$ \\
\hline \multirow[t]{2}{*}{$\begin{array}{l}\text { Detection } \\
\text { bias }\end{array}$} & $\begin{array}{l}\text { Controlled } \\
\text { studies }\end{array}$ & $\begin{array}{l}\text { High risk if personnel evaluating } \\
\text { outcomes were not blinded }\end{array}$ \\
\hline & $\begin{array}{l}\text { Observational } \\
\text { studies }\end{array}$ & $\begin{array}{l}\text { High risk if there were systematic } \\
\text { differences in outcomes } \\
\text { assessment among comparison } \\
\text { groups } \\
\text { High risk if the measurement of } \\
\text { exposure is flawed, for example, } \\
\text { recall bias in case-control studies }\end{array}$ \\
\hline
\end{tabular}

\begin{tabular}{lll}
\hline Type of bias & Type studies & Low/High risk \\
\hline $\begin{array}{l}\text { Reporting } \\
\text { bias }\end{array}$ & $\begin{array}{l}\text { Controlled } \\
\text { studies }\end{array}$ & $\begin{array}{l}\text { High risk if reporting of outcomes } \\
\text { is not prespecified as of interest } \\
\text { to the review }\end{array}$ \\
$\begin{array}{l}\text { Observational } \\
\text { studies }\end{array}$ & $\begin{array}{l}\text { High risk if there are systematic } \\
\text { differences between reported and } \\
\text { unreported findings }\end{array}$ \\
$\begin{array}{ll}\text { Confounding } \\
\text { Controlled } \\
\text { studies }\end{array}$ & $\begin{array}{l}\text { Low risk if allocation was } \\
\text { balanced between groups by for } \\
\text { example, matching, stratification, } \\
\text { etc }\end{array}$ \\
& $\begin{array}{l}\text { Observational } \\
\text { studies }\end{array}$ & $\begin{array}{l}\text { High risk, but can be mitigated } \\
\text { using matching by propensity } \\
\text { scores, etc }\end{array}$ \\
& $\begin{array}{l}\text { High risk if there is a failure to } \\
\text { adjust for important confounders } \\
\text { in the statistical analysis. }\end{array}$ \\
\hline
\end{tabular}

\section{Confounders relevant to all or most of the studies}

Important confounding factors in this study are those that affect the association of antimicrobial exposure and organ injury among elderly patients. The severity of the disease being treated, comorbidities and non-antimicrobial medication known to risk organ injury will be considered as significant confounders. The disease severity will be determined using a validated tool such as the Acute Physiology and Chronic Health Evaluation tool. ${ }^{16}$ Sex and age will also be considered a priori. Included studies will be assessed on whether they adjusted for these confounders, and any other additional confounders such as dose adjustment and administration route, to determine their final outcomes or not.

\section{Strategy for data synthesis \\ Descriptive analysis of data}

All studies that meet the inclusion criteria will be described, including the following:

1. Study design-including the study quality, data collection methods, the validity of tools used and the statistical analysis.

2. Participants (both exposed and unexposed)-including demographic and socio-economic characteristics and health status such as the severity of the disease.

3. Exposure-the types of antimicrobials used, duration and frequency of use and spectrum (broad or narrow) of effectiveness.

4. Outcomes-primary and secondary outcomes for each study will be described.

\section{Statistical analysis}

Meta-analysis for quantitative data will be carried out for only those observational studies scoring at least six on the Newcastle-Ottawa scale. The analysis will be done using the more recent version of the $\mathrm{R}$ statistical software ( $\mathrm{R}$ i386 4.0.2). Results for studies that show enough homogeneity will be pooled together using the random-effects model. Relative risks, calculated at 95\% CIs and two-sided $p$ values, will be used as the primary effect measure. If homogeneity is insufficient, quantitative results will be 
tabulated and a narrative synthesis will be provided to summarise the results displayed in the included studies. Heterogeneity among the observational studies will be assessed using the Cochran's $Q$ test ${ }^{17}$ at $5 \%$ significance level and quantified using the $\mathrm{I}^{2}$ statistic. ${ }^{17}$ An $\mathrm{I}^{2}$ value of more than $50 \%$ will be considered to reflect substantial heterogeneity and therefore will trigger sensitivity analysis to investigate the possible source of heterogeneity. For the results of the meta-analysis, publication bias will be assessed using funnel plot. ${ }^{18}$ Details of each included study will also be presented in a table of study characteristics, from which exploratory descriptive analyses will be done.

\section{Analysis of subgroups or subsets}

Subgroup analysis by age (65-80 years and more than 80 years), sex, ethnicity, type of organ damage and antimicrobial class will be carried out. Additional subgroups will be analysed but it is not easy to specify them in advance.

\section{Patient and public involvement}

No patient involved.

\section{Strategy for the presentation of results}

For each database used, the final search strategy, and any other additional searches done, will be provided in a different file. The process of selecting articles from title screening up to final article inclusion into the final review will be shown on a flow diagram following the PRISMA guidelines. ${ }^{7}$ Information about the rationale of exclusion during full article screening will be presented in this flow diagram. A funnel plot will be used to demonstrate any potential small study effects and potential publication bias for any meta-analysis with at least six studies. ${ }^{19}$ The characteristics of studies tables and meta-analyses results of this review will be compiled into a publishable journal article. Other information will be included by text, descriptive statistics and a summary table of findings. The GRADE tool ${ }^{15}$ will be used to guide the construction of the summary of findings table. The full results on the quality of individual study assessment using the Newcastle-Ottawa scale will be provided in the additional file. However, the summarised table of these findings will be presented in the main article.

\section{Ethical considerations}

This systematic review and meta-analysis will not involve directly working with patients or human tissues. However, during the conduct of the review, ethical principles will still be observed to ensure integrity. Any potential conflicts of interests will be declared, all contributors acknowledged and no plagiarised material will be included in the review. ${ }^{20}$

\section{DISSEMINATION}

The systematic review and meta-analysis will be submitted for publication in a peer-reviewed journal in geriatrics. The findings will be presented at international conferences in the area of geriatrics or pharmacoepidemiology. This work will contribute to a PhD undertaken at the University of Bath. The results will be communicated to patient and public engagement networks supported by the National Health Service (NHS) Research and Development office, Bath, Swindon and Wiltshire.

\section{DISCUSSION}

This protocol describes the method for the synthesis of current evidence related to antimicrobial exposure and organ injury among older adults aged 65 years or more. The proposed systematic review will probably be the first to summarise the association of antimicrobial use and organ injury among the older adults. We anticipate significant heterogeneity between the included studies, and we may not have enough studies to pool together to conduct a meta-analysis for each of the primary outcomes of interest. However, we may be able to generate evidence on the risk of antimicrobial exposure on organ injury as a composite measure. A clearer understanding of this relationship will unravel any information gaps, advance academic literature and inform the development of antimicrobial prescribing policies for this vulnerable population.

Acknowledgements The authors would like to thank Linda Humphreys, the science faculty librarian, University of Bath, for here immense help in training and support on article search strategies from various databases.

Contributors TC will serve as the first author of the protocol and the review paper. He led all the stages of the development of this protocol, while PSN and SR supervised and contributed to the development of plans for searching, screening, extracting and writing phases.

Funding The authors have not declared a specific grant for this research from any funding agency in the public, commercial or not-for-profit sectors.

Competing interests None declared.

Patient consent for publication Not applicable.

Provenance and peer review Not commissioned; externally peer reviewed.

Supplemental material This content has been supplied by the author(s). It has not been vetted by BMJ Publishing Group Limited (BMJ) and may not have been peer-reviewed. Any opinions or recommendations discussed are solely those of the author(s) and are not endorsed by BMJ. BMJ disclaims all liability and responsibility arising from any reliance placed on the content. Where the content includes any translated material, BMJ does not warrant the accuracy and reliability of the translations (including but not limited to local regulations, clinical guidelines, terminology, drug names and drug dosages), and is not responsible for any error and/or omissions arising from translation and adaptation or otherwise.

Open access This is an open access article distributed in accordance with the Creative Commons Attribution Non Commercial (CC BY-NC 4.0) license, which permits others to distribute, remix, adapt, build upon this work non-commercially, and license their derivative works on different terms, provided the original work is properly cited, appropriate credit is given, any changes made indicated, and the use is non-commercial. See: http://creativecommons.org/licenses/by-nc/4.0/.

ORCID iD

Tichawona Chinzowu http://orcid.org/0000-0002-4007-4974

\section{REFERENCES}

1 Augustine S, Bonomo RA. Taking stock of infections and antibiotic resistance in the elderly and long-term care facilities: a survey 
of existing and upcoming challenges. Eur J Microbiol Immunol 2011;1:190-7.

2 Esme M, Topeli A, Yavuz BB, et al. Infections in the elderly critically-ill patients. Front Med 2019;6:118

3 Giarratano A, Green SE, Nicolau DP. Review of antimicrobial use and considerations in the elderly population. Clin Interv Aging 2018;13:657-67.

4 Le J. MSD manual. professional version. drug excretion, 2020. Available: https://www.msdmanuals.com/en-gb/professional/clinicalpharmacology/pharmacokinetics/drug-excretion?query=renal\% 20elimination\%20of\%20drugs

5 Ruscin JMaL SA. MSD manual professional version. pharmacokinetics in older adults, 2021. Available: https://www. $\mathrm{msdmanuals.com/professional/geriatrics/drug-therapy-in-older-}$ adults/pharmacokinetics-in-older-adults

6 Werth BJ. MSD manual professional version. fluoroquinolones, 2020. Available: https://www.msdmanuals.com/professional/infectiousdiseases/bacteria-and-antibacterial-drugs/fluoroquinolones?query= antibiotic\%20associated\%20tendonitis

7 Moher D, Liberati A, Tetzlaff J, et al. Preferred reporting items for systematic reviews and meta-analyses: the PRISMA statement. BMJ 2009;339:b2535.

8 Moher D, Shamseer L, Clarke M, et al. Preferred reporting items for systematic review and meta-analysis protocols (PRISMA-P) 2015 statement. Syst Rev 2015;4:1.

9 Ranganathan P, Pramesh CS, Aggarwal R. Common pitfalls in statistical analysis: absolute risk reduction, relative risk reduction, and number needed to treat. Perspect Clin Res 2016;7:51-3.
$10 \mathrm{Kim} \mathrm{H}$-Y. Statistical notes for clinical researchers: risk difference, risk ratio, and odds ratio. Restor Dent Endod 2017;42:72-6.

11 Web of Science. Endnote: endnote X9. Available: https://clarivate. libguides.com/endnote_training/users/enx9

12 Covidence. Bringing the best evidence into practice faster. Available: https://www.covidence.org/reviewers/

13 Sterne JAC, Savović J, Page MJ, et al. Rob 2: a revised tool for assessing risk of bias in randomised trials. BMJ 2019;366:14898.

14 et alWells GA SB, O'Connell D, Peterson J. The Newcastle-Ottawa scale (NOS) for assessing the quality of nonrandomised studies in meta-analyses. Available: http://www.ohri.ca/programs/clinical_ epidemiology/oxford.asp

15 Atkins D, Best D, Briss PA, et al. Grading quality of evidence and strength of recommendations. BMJ 2004;328:1490.

16 Knaus WA, Draper EA, Wagner DP, et al. Apache II: a severity of disease classification system. Crit Care Med 1985;13:818-29.

17 Higgins JPT, Thompson SG, Deeks JJ, et al. Measuring inconsistency in meta-analyses. BMJ 2003;327:557-60.

18 Sterne JA, Egger M. Funnel plots for detecting bias in metaanalysis: guidelines on choice of axis. J Clin Epidemiol 2001;54:1046-55.

19 Higgins JPT, Altman DG, Gøtzsche PC, et al. The Cochrane collaboration's tool for assessing risk of bias in randomised trials. BMJ 2011;343:d5928.

20 Wager E, Wiffen PJ. Ethical issues in preparing and publishing systematic reviews. J Evid Based Med 2011;4:130-4. 